\title{
Primary squamous cell carcinoma of the liver: an unexpected pathological finding
}

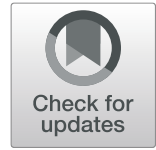

\author{
Fabio Tuminello ${ }^{1}$, Davide Castiglione ${ }^{2}$, Giuseppe Broggi ${ }^{3}$, Giada Maria Vecchio ${ }^{3}$, Antonio Basile², \\ Stefano Puleo ${ }^{1}$ and Antonio Pesce ${ }^{1 *}$
}

\begin{abstract}
Background: Primary squamous cell carcinoma of the liver is a very rare cancer requiring a complete diagnostic investigation, as it presents some differences from other primary hepatic malignancies. We describe the case of an elderly patient with primitive squamous cell carcinoma of the liver treated by percutaneous microwave ablation and transarterial chemoembolization.

Case presentation: Although the pathogenesis remains uncertain, a possible transformation of the biliary epithelium caused by chronic inflammation or metaplasia and subsequent malignant transformation of preexisting benign hepatic cysts has been suggested. A critical review of the existing literature was performed by focusing on the pathogenesis, clinical presentation, and the suggested therapeutic strategies.

Conclusion: A multidisciplinary approach is needed to face rare hepatic malignancies where there are no clear guidelines.
\end{abstract}

Keywords: Squamous cell carcinoma, Liver cyst, Metaplasia, Transarterial embolization, Multidisciplinary approach

\section{Background}

Primary squamous cell carcinoma (SCC) of the liver is a very uncommon finding in everyday clinical practice. The most common anatomical areas are represented by the skin, lungs, distal esophagus, anal canal, cervical, or inguinal lymph nodes, in which a squamocellular epithelium may be identified. Primary SCC of the liver has been mostly reported to be sporadic and associated with hepatic cysts, hepatolithiasis, or hepatic teratoma [1-3].

Although the pathogenesis remains uncertain, a possible transformation of the biliary epithelium caused by chronic inflammation or metaplasia and subsequent malignant transformation of preexisting benign hepatic cysts has been suggested [4-6].

\footnotetext{
* Correspondence: nino.fish@hotmail.it

This case report was presented at the following Italian Society of Surgical Researches (SIRC) meeting: «Chirurgia Insieme: Convegno Congiunto delle Società Specialistiche Chirurgiche Italiane», Genoa 27-28 June 2019

'Section of General Surgery, Department of Medical, Surgical Sciences and Advanced Technologies "G.F. Ingrassia", University Hospital

"Policlinico-Vittorio Emanuele", 95123 Catania, Italy

Full list of author information is available at the end of the article
}

Because of its rare prevalence, the histopathological analysis is imperative and it is often a diagnosis of exclusion, after having carefully examined the patient, looking for other possible primary lesions and ruling out the possibility for secondary SCC.

The prognosis of this rare tumor of the liver is extremely poor and only few patients survive more than 12 months even after surgical resection.

Herein, we reported a case of an elderly patient with primitive squamous cell carcinoma of the liver treated by percutaneous microwave ablation and transarterial embolization. A critical review of the existing literature was performed by focusing on the pathogenesis, clinical presentation, and the suggested therapeutic strategies.

\section{Case presentation}

A 79-year-old Caucasian man presented with profuse asthenia, unintentional weight loss (around $20 \mathrm{~kg}$ ) over the last 3 months, and abdominal chronic pain in the right hypochondrium. He denied alcohol abuse and smoking. The past medical history reported essential hypertension 
under pharmacological treatment, sigmoid diverticulosis, and cholelithiasis. Physical examination revealed a treatable abdomen, aching on the deep palpation in the right hypochondrium, where the inferior margin of the liver was appreciated $5 \mathrm{~cm}$ below the costal arch and a hard irregular mass was recognized. No cervical, axillary, or inguinal lymphadenopathy were appreciated. A dermatological counselling excluded skin lesions. Digital rectal examination was negative. Breathing sound was clear without wheezing or crackle. The remaining physical examination was normal.

Blood tests showed ALT $91 \mathrm{U} / \mathrm{l}, \mathrm{GGT} 308 \mathrm{U} / \mathrm{l}$, total bilirubin level $1.7 \mathrm{mg} / \mathrm{dl}$, Ca $19-91956 \mathrm{U} / \mathrm{ml}$, AFP 1.6 $\mathrm{ng} / \mathrm{ml}$, CEA $12 \mathrm{U} / \mathrm{ml}$.

Upper endoscopy and colonoscopy excluded esophago-gastric and colorectal lesions, respectively. Abdominal computed tomographic scan (CT-scan) showed a large hypodense liver mass in the right lobe $(15 \mathrm{~cm} \times$ $12 \mathrm{~cm}$ in size) on both arterial and portal phases involving the IV-V-VI-VII-VIII segments (Fig. 1a,b) which presented colliquative areas of necrosis within the lesion. Mild dilatation of intrahepatic biliary ducts was observed. Multiple isolated liver cysts were described in both lobes. Moreover, the lesion was very close to the gallbladder, which showed wall thickening and enhanced endoluminal tissue of uncertain origin. The cystic duct was enlarged, and focal endoluminal tissue was detected. Some enlarged lymph nodes in the right subdiaphragmatic space were reported.

The radiologist, based on these findings, hypothesized the suspicion of gallbladder cancer or intrahepatic cholangiocarcinoma mass forming. A liver biopsy was performed under local anesthesia via combined US/CT guidance. The interventional radiologist used core needle biopsy device (Monopty $18 \mathrm{G} \times 16 \mathrm{~cm}$, BARD, Arizona, USA). Two specimens were collected. No complication occurred.

Histopathological analysis of the liver mass showed a well-differentiated squamous cell carcinoma with diffuse necrosis. Immunohistochemical staining was positive for cytokeratins (CK) 7, 19, and p63; anti-hepatocyte, MUC5 , TTF-1 were negative, suggesting the tumor as primary or metastatic squamous cell carcinoma of the liver (Figs. $2 \mathrm{a}-\mathrm{d}$ and 3$)$.

After the surprising and unexpected pathological report, a PET/CT scan was performed in order to rule out the presence of another primitive unknown tumor. The examination showed a single area of increased uptake in the right lobe of the liver, corresponding to the tumor mass. The uptake was inhomogeneous with the central part of the mass showing weak or no uptake and the peripheral regions showing an intense uptake. This finding could confirm the presence of areas of colliquative necrosis and areas of active neoplasm, as shown in Fig. 4. No other anatomic regions of altered metabolic captation were discovered. Therefore, the diagnosis of primary squamous cell carcinoma of the liver was confirmed.

After a multidisciplinary meeting involving surgeons, radiologists, oncologists, and hepatologists, the case was accurately discussed and the different treatment options were evaluated. The patient was initially scheduled for major liver resection (extended right hepatectomy after portal venous embolization), but he refused it. In consideration of the size of the mass and the paucity of scientific recommendations, a mini-invasive radiological approach was considered appropriate. A combined approach was proposed: percutaneous transhepatic microwave ablation and transarterial chemoembolization (TACE).

On the same day, the interventional radiology team performed microwave ablation via US guidance, with two different probes at $100 \mathrm{~W}$ of energy for $9 \mathrm{~min}$ (Evident $^{\text {tim }}$ MW Ablation System, Medtronic, Minneapolis, MN, USA) and transarterial chemoembolization (TACE). TACE was performed via left radial artery access, using Simmons 1 catheter and hydrophilic guidewire to negotiate the celiac axis and the proper hepatic artery. Unfortunately, celiac axis dissection was observed during angiographic study and the procedure was

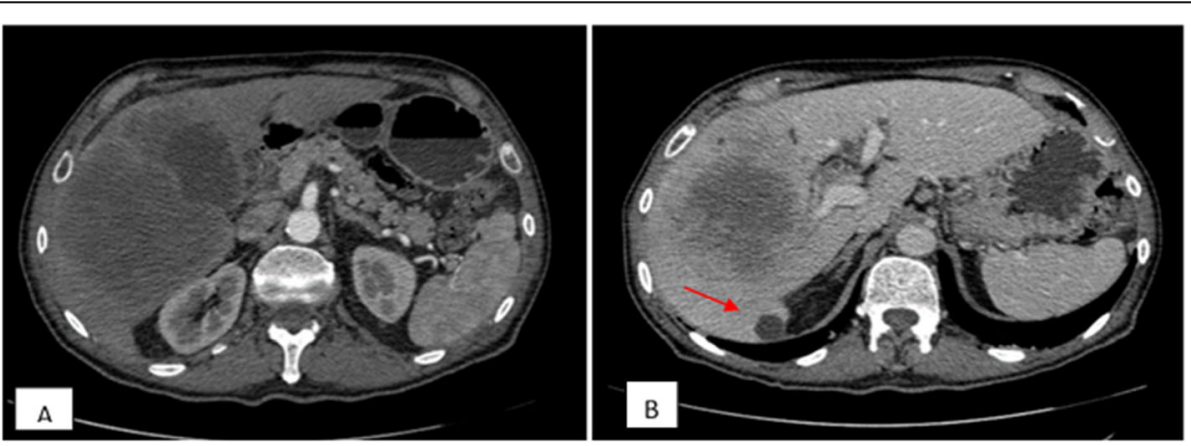

Fig. 1 a Abdominal CT scan, axial view, arterial phase, showing hypodense mass in the right lobe of the liver with weakly enhanced margin. $\mathbf{b}$ Portal phase, hypodense liver mass and the presence of hepatic cyst (red arrow). Mild dilation of intrahepatic biliary tree 

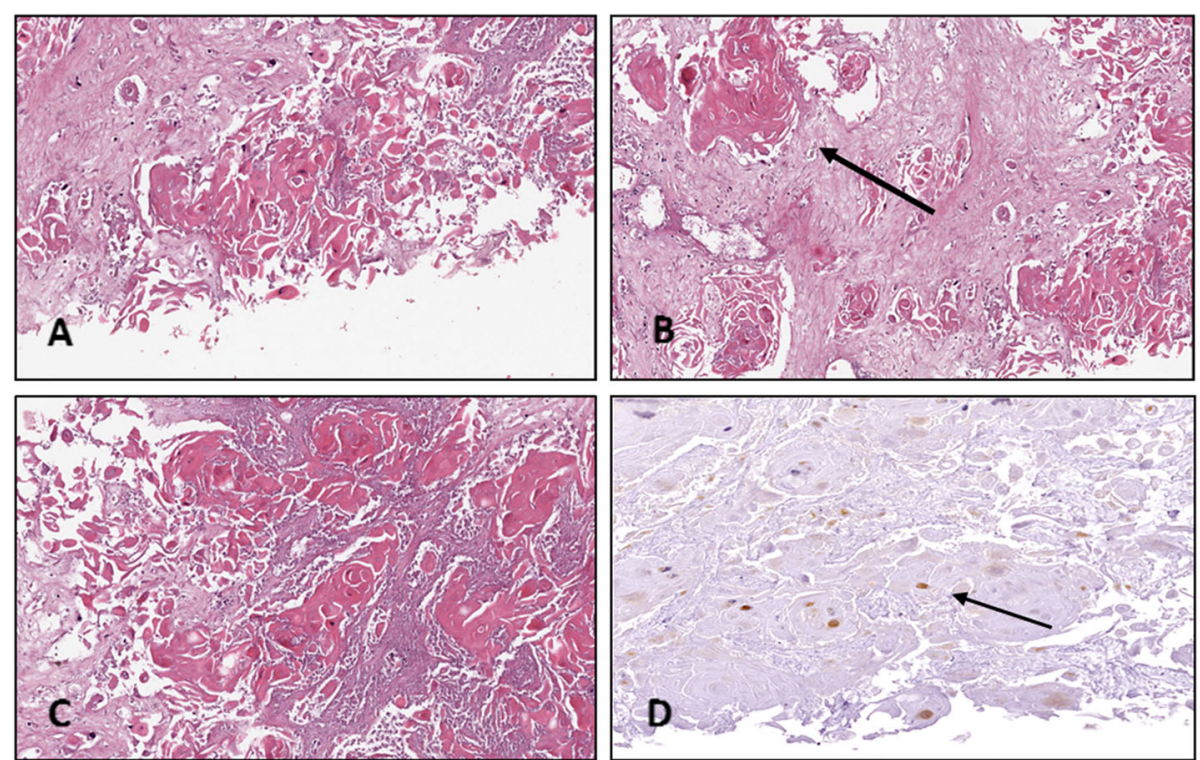

Fig. 2 a Showing a well-differentiated squamous cell carcinoma $(H \& E, \times 100)$. b The tumor shows obvious squamous cell features such as seen here (arrow). c Well-differentiated lesion shows prominent keratinization and may form "pearl-like" structures (40 X). Interposed areas of necrosis and fibrosis are evident. d Diffuse nuclear staining (arrow) for p63 in squamous neoplastic cells

completed negotiating the hepatic artery via collateral hypertrophic vessels arising from the superior mesenteric artery. Arteriography of the right hepatic artery showed no relevant hypervascular mass as expected. Some anarchic and tortuous arteries were confirmed as tributaries of the mass (Fig. 5) and then embolized with microparticles $(150-300 \mu \mathrm{m})$. The immediate postprocedural course was uneventful. The patient presented a global improvement of his quality of life in terms of abdominal pain relief. He was discharged with a poor prognosis.

At 1-month follow-up, an abdominal CT scan did not show significant differences, After the sixth month, the patient developed obstructive jaundice with significant

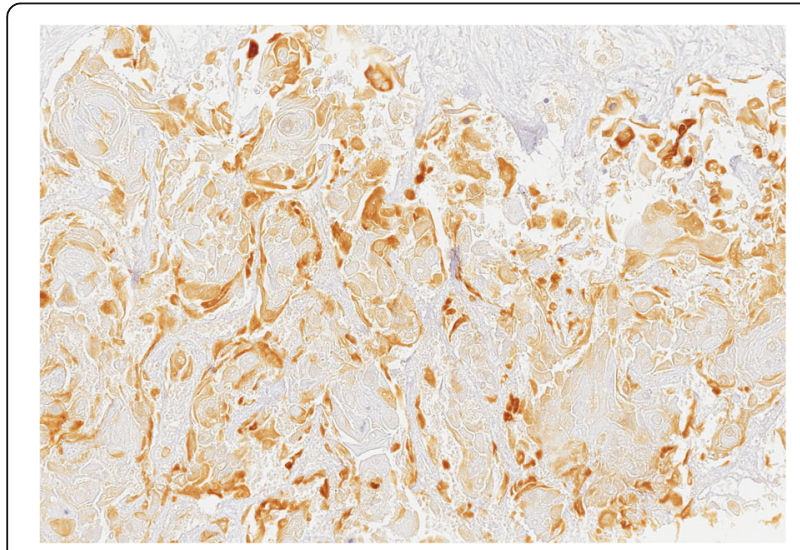

Fig. 3 Showing diffuse positive staining for CK19 in squamous tumor cells dilatation of intrahepatic biliary system; the liver mass had grown and a percutaneous transhepatic biliary drainage was put in.

He died 9 months later.

\section{Discussion}

Primary SCC of the liver is a very rare cancer requiring a complete diagnostic investigation, as it presents some differences from other primary hepatic malignancies [79]. In a recent review, Zhang XF et al. [3] reported only 31 cases described in English literature since 1970s and other four patients encountered in their study. The main risk factors associated with the occurrence of SCC of the liver are male sex, hepatic cysts, hepatolithiasis, and liver cirrhosis.

Various etiopathogenetic theories have been proposed to explain the origin of primary squamous cell carcinoma of the liver. Some authors suggest that primary SCC of the liver is often associated with hepatic cysts, but its exact path to carcinoma is not yet proven. Continuous irritation due to chronic inflammation might promote secondary squamous metaplasia and subsequent malignant transformation. In the current case, the patient presented hepatolithiasis and multiple benign liver cysts which may explain the possible trasformation of cyst epithelium to squamocellular carcinoma, but the causes of carcinogenesis need to be further studied. It may be very interesting to establish animal models to mimic the malignant transformation of cyst epithelium to squamous carcinomatous epithelium. In some patients, an 

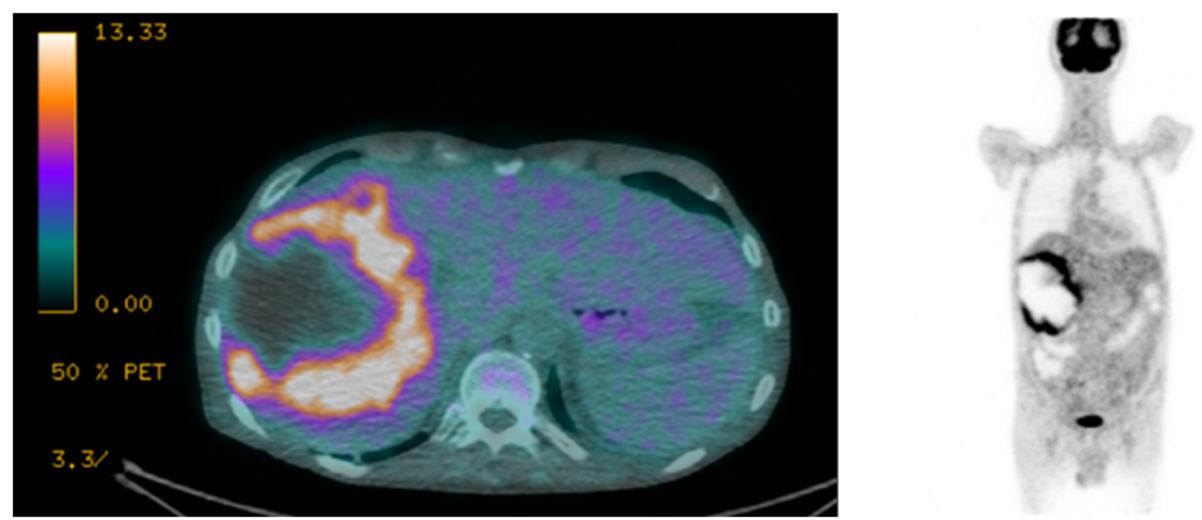

Fig. 4 PET imaging, fusion PET/CT, and PET coronal view showing the high uptake of liver mass. The difference between inner and outer part of the mass are highlighted

association of adenomatous and squamous components has been reported in English literature [10-13].

According to many studies [2-4], patients with primary SCC of the liver may present various symptoms including chronic abdominal pain or general discomfort, anorexia, weight loss, jaundice, and rarely progressive dysphagia. Our patient presented chronic pain in the right hypochondrium, asthenia, remarkable weight loss, and only after a couple of months, he developed obstructive jaundice.

The delayed appearance of jaundice has been an interesting matter of discussion: we considered it as a possible biological characteristic of this particular kind of tumor, with important differences with gallbladder cancer and intrahepatic cholangiocarcinoma, for example.

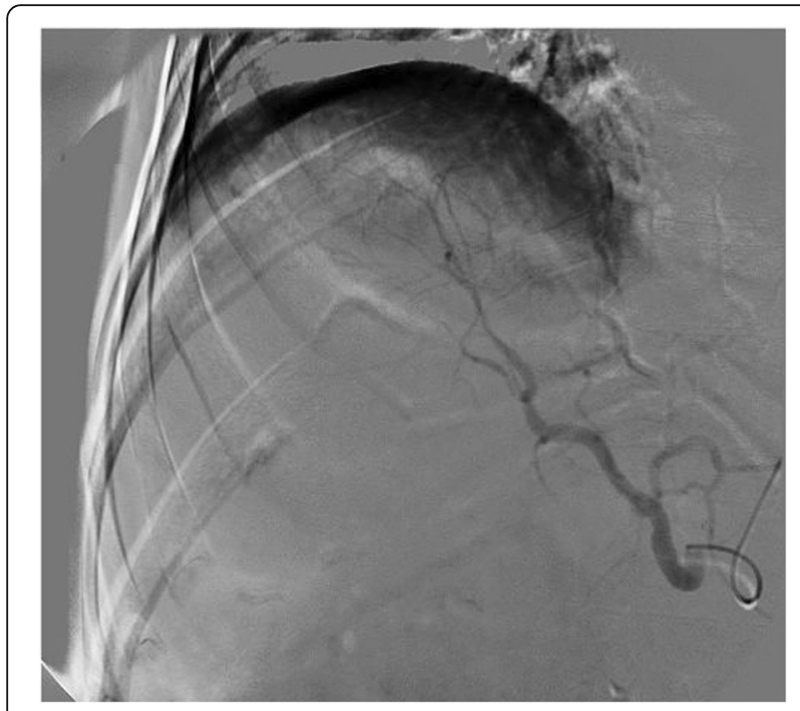

Fig. 5 Digital subtraction angiography (DSA) shows Cobra C2 5Fr catheter in mesenteric artery branch. Accessory right hepatic artery, increased in diameter, acts as anarchic vascularization towards the liver tumor.
Most of these patients may present increased serum levels of ALT, AST, and even bilirubin, due to chronic inflammation in bile ducts or liver cyst and tumor progression. However, there is a lack of specific serum marker of primary liver SCC at the present time.

Sometimes, the clinical and radiological diagnosis is quite hard. Radiological investigations may present important pitfalls. CT seems to be the most valuable preoperative investigation. After systematically examining all anatomical districts from which it can originate, the diagnosis of primary SCC of the liver should only be considered when a suitable biopsy specimen documents the presence of squamous cells. Immunohistochemistry plays an important diagnostic role. The strong positive staining for CK 14 and CK 56 suggest basal cells of keratinized squamous epithelium origin of the cancer cells. Moreover, the positive expression of CK19 confirms the bile ductular ontogeny of the neoplastic cells.

There are still no clear guidelines on the treatment of primary SCC of the liver, unlike other primitive hepatic malignancies in which there are clear scientific recommandations [14-17]. Therapeutic options include surgical resection, chemotherapy, and radiotherapy. Transarterial chemoembolization (TACE) has been reported for treatment of primary SCC of the liver [3]. Like most of the hepatic malignancy, surgical resectability represents the most important factor for improving the prognosis. According to Zhang XF et al. [3], patients undergoing radical surgery had longer overall survival time than those undergoing palliative treatment (median survival 17 vs 5 months, $p=0.005$ ). Therefore, complete surgical excision of the tumor is strongly recommended rather than simple drainage or partial excision. Systemic or intra-arterial chemotherapy might be useful in surgically unfit patients $[7,18,19]$. In our specific case, the patient was initially scheduled for major liver resection but after refusing surgical option, a radiological combined approach was preferred to systemic chemotherapy 
or radiotherapy, with acceptable prognosis in terms of quality of life. The prognosis of squamous cell carcinoma of the liver is extremely poor, with a median overall survival of a few months. However, Kaji R et al. [18] described a case of SCC of the liver that remarkably responded to low-dose intra-hepatoarterial chemotherapy with overall survival of 23 months.

\section{Conclusion}

A multidisciplinary approach is needed to face rare hepatic malignancies where there are no clear guidelines in order to choose the best therapeutic option for patients.

\section{Abbreviations}

SCC: Primary squamous cell carcinoma; ALT: Alanine aminotransferase; AST: Aspartate aminotransferase; GGT: Gamma-glutamyltransferase; AFP: Alpha fetoprotein; TTF-1: Transcriptional thyroid factor 1;

TACE: Transarterial chemoembolization; CEA: Carcinoembryonic antigen; CA 19-9: Cancer antigen 19-9

\section{Acknowledgements}

Not applicable.

\section{Authors' contributions}

All authors contributed equally to the paper; FT, AP, and SP conceptualized and designed the case report; FT drafted the initial manuscript; $D C$ and $A B$ performed interventional radiology treatment; GB and GMV perfomed histological analysis. All authors reviewed and approved the final version of the manuscript as submitted.

\section{Funding}

No funding

\section{Availability of data and materials}

The data and material related to this case report are available.

\section{Ethics approval and consent to participate}

This study was exempted from ethical approval in our institution.

\section{Consent for publication}

Written informed consent was obtained from the patient for publication of this case report and accompanying images.

\section{Competing interests}

The authors declare that they have no competing interests.

\section{Author details}

${ }^{1}$ Section of General Surgery, Department of Medical, Surgical Sciences and Advanced Technologies "G.F. Ingrassia", University Hospital "Policlinico-Vittorio Emanuele", 95123 Catania, Italy. ${ }^{2}$ Radiology I Unit, Department of Medical, Surgical Sciences and Advanced Technologies "G.F. Ingrassia", University Hospital "Policlinico-Vittorio Emanuele", 95123 Catania, Italy. ${ }^{3}$ Anatomic Pathology, Department of Medical, Surgical Sciences and Advanced Technologies "G.F. Ingrassia", University Hospital

"Policlinico-Vittorio Emanuele", 95123 Catania, Italy.

Received: 19 November 2019 Accepted: 19 March 2020

Published online: 26 May 2020

\section{References}

1. Bloustein PA, Silverberg SG (1976) Squamous cell carcinoma originating in a hepatic cyst. Case report with a review of the hepatic cystcarcinoma association. Cancer. 38(5):2002-2005

2. Odemiş B, Köksal AS, Yüksel O, Kacar S, Turhan N (2006 Jul) Squamous cell cancer of the liver arising from an epidermoid cyst: case report and review of the literature. Dig Dis Sci. 51(7):1278-1284
3. Zhang XF, Du ZQ, Liu XM, Lv Y (2015 Jul) Primary squamous cell carcinoma of liver - case series and review of literatures. Medicine (Baltimore). 94(28): e868

4. Weimann A, Klempnauer J, Gebel M, Maschek H, Bartels M, Ringe B, Pichlmayr R (1996) Squamous cell carcinoma of the liver originating from a solitary non-parasitic cyst case report and review of the literature. HPB Surg. 10(1):45-49

5. Nakajima T, Kondo Y (1990 Mar 15) A clinicopathologic study of intrahepatic cholangiocarcinoma containing a component of squamous cell carcinoma. Cancer. 65(6):1401-1404

6. Yoo TK, Kim Bl, Han EN, Kim DH, Yoo JH, Lee SJ, Cho YK, Kim HJ (2016 Mar) Primary squamous cell carcinoma of the liver: a case report. Clin Mol Hepatol. 22(1):177-182

7. Boscolo G, Jirillo A, Da Pian P (2005 Jan-Feb) Complete remission of poorly differentiated squamous liver carcinoma after systemic chemotherapy and surgery. A case report. Tumori. 91(1):71-72

8. Wilson JM, Groeschl R, George B, Turaga KK, Patel PJ, Saeian K, Gamblin TC (2013) Ciliated hepatic cyst leading to squamous cell carcinoma of the liver - a case report and review of the literature. Int J Surg Case Rep. 4(11):972975

9. Morito K, Kai K, Miyoshi A, Kubo H, Ide T, Azama S, Irie H, Koga Y, Sumi K, Ikeda O, Kitahara K, Noshiro H (2013 Apr) Primary squamous cell carcinoma of the liver concomitant with primary colon cancer: report of a case. Clin J Gastroenterol. 6(2):134-138

10. Harino T, Tomimaru Y, Noguchi K, Nagase H, Ogino T, Hirota M, Oshima K, Tanida T, Noura S, Imamura H, Akagi K, Iwazawa T, Tamura H, Adachi S, Dono K (2019 Apr) A rare case of adenosquamous carcinoma in the liver with hepatolithiasis. Gan To Kagaku Ryoho. 46(4):772-774

11. Tamaoka K, Tanemura M, Furukawa K, Mikamori M, Saito T, Ohtsuka M, Suzuki Y, Tei M, Kishi K, Yasuoka H, Tsujimoto M, Akamatsu H (2018 Oct 5) Primary intrahepatic squamous cell carcinoma with histological collision of adenocarcinoma and squamous cell carcinoma: a case report. Am J Case Rep. 19:1184-1191

12. Yamao K, Takenaka M, Imai H, Nakai A, Omoto S, Kamata K, Minaga K, Miyata T, Sakurai T, Watanabe T, Nishida N, Matsumoto I, Takeyama Y, Chikugo T, Kudo M (2017) Primary hepatic adenosquamous carcinoma associated with primary sclerosing cholangitis. Oncology. 93(Suppl 1):76-80

13. Gao S, Chen D, Huang L, Wu L, Dai R, Shan Y (2015 Aug 1) Primary adenosquamous carcinoma of the liver: a case report and review of the literature. Int J Clin Exp Pathol. 8(8):9687-9692

14. Trovato MA, Pesce A, Sofia M, Montineri A, Basile A, Palermo F, Fatuzzo F, Nigro L, Puleo S (2013 Oct) Is BCLC algorithm useful in clinical practice? Study on 164 HCC patients. Hepatogastroenterology. 60(127):1742-1745

15. Pesce A, Scilletta R, Branca A, Nigro L, Montineri A, Larocca L, Fatuzzo F, Castaing M, Puleo S (2012 Jun) Does transient elastography (FibroScan ${ }^{\circledast}$ ) have a role in decision making in hepatocellular carcinoma? HPB (Oxford). 14(6):403-408

16. Aloia TA, Járufe $N$, Javle M, Maithel SK, Roa JC, Adsay V, Coimbra FJ, Jarnagin WR (2015 Aug) Gallbladder cancer: expert consensus statement. HPB (Oxford). 17(8):681-690

17. Weber SM, Ribero D, O'Reilly EM, Kokudo N, Miyazaki M, Pawlik TM (2015 Aug) Intrahepatic cholangiocarcinoma: expert consensus statement. HPB (Oxford). 17(8):669-680

18. Kaji R, Sasaki N, Tateishi I, Nagata E, Okabe Y, Yoshida T, Sata M, Ueno T (2003) A case report of primary hepatic squamous cell carcinoma that remarkably responded to low dose arterial injection of anti-cancer drugs. Kurume Med J. 50:71-75

19. Naik S, Waris W, Carmosino L, Mehrishi A, Saif MW (2009) Primary squamous cell carcinoma of the liver. J Gastrointestin Liver Dis. 18:487-489

\section{Publisher's Note}

Springer Nature remains neutral with regard to jurisdictional claims in published maps and institutional affiliations. 